Aden, Joëlle, et Sandrine Eschenauer. 2020. « Une pédagogie enactive-performative de la translangageance en milieu plurilingue ». P. 177-99 in Perspektiven auf Mehrsprachigkeit im Fremdsprachenunterricht - Regards croisés sur le plurilinguisme et l'apprentissage des langues, Literatur-, Kultur- und Sprachvermittlung: LiKuS, édité par B. Schädlich. Berlin, Heidelberg: Metzler/Springer.

Pr. Dr. habil. Joëlle Aden

Sciences du langage, CNU 7è section

Université Paris-Est Créteil (UPEC)

IMAGER EA 3958/ LIDIL12

joelle.aden@u-pec.com

Dr. Sandrine Eschenauer

Sciences du langage, CNU 7è section

Aix-Marseille Université

Laboratoire Parole et Langage (LPL UMR 7309, CNRS et AMU)

Institut National Supérieur du Professorat et de l'Education (INSPE) \& Institut de la Créativité et de

l'Innovation (InCIAM)

\title{
Une pédagogie enactive-performative de la translangageance en milieu plurilingue
}

Résumé : Dans cet article, nous définissons le «translangager » dans le paradigme de l'enaction en intégrant les dimensions kinesthésique et émotionnelle du langage à la notion de compétence plurilingue. Nous expliquons ensuite en quoi notre approche performative constitue une pédagogie du tranlangager ; pour cela, nous mettons en regard le champ des "performance studies" et celui de l'enaction. Il s'agit de montrer qu'au-delà d'une compétence communicative, ce processus holistique et multimodal que nous nommons "translangageance" est une fonction constitutive de la connaissance qui émerge dans l'interaction. Nous concluons par la présentation d'une étude longitudinale qui vise à entraîner la compétence de translangageance dans une classe bilangue en milieu plurilingue.

Mots clé: énaction, créativité, approches performatives, didactique des langues, translanguaging

Resümee: In diesem Artikel definieren wir das "Transsprechen" im Rahmen des Enaktionsparadigmas. Wir betrachten dabei die kinästhetische und emotionale Dimension der Sprache als Teil der Mehrsprachigkeitskompetenz. Es wird daraufhin erklärt, auf welche Weise unser performativer Ansatz eine Pädagogik des Transsprechens verwirklicht. Zu diesem Zweck werden die Wechselbeziehungen zwischen dem Forschungsfeld der "Performance Studies" und dem der Enaktion aufgezeigt. Es soll dargestellt werden, dass der ganzheitliche und multimodale Prozess, den wir "translangageance" nennen, eine grundlegende Funktion der Erkenntnis ausmacht, welche selbst in der Interaktion entsteht. Abschließend stellen wir eine Langzeitstudie vor, die zum Ziel hatte, die Translangageance als Kompetenz in einer "classe bilangue" (zwei Fremdsprachen gleich zu Beginn der Sekundarstufe 1) im mehrsprachigen Milieu zu fördern.

Schlüsselwörter: Enaction, Kreativität, performative Ansätze, Sprachdidaktik, Translanguaging

\section{Introduction}


«L'acte de communiquer (se traduit par) le modelage mutuel d'un monde commun au moyen d'une action conjuguée : c'est notre réalisation sociale, par l'acte de langage, qui prête vie à notre monde. » (Varela, 1988, p. 115)

Dans cet article, nous présentons notre approche pédagogique du plurilinguisme élaborée à la croisée du paradigme de l'enaction de Francisco Varela (1989, 1993, 1999a, 1999b) et du courant performatif (Austin, 1962 ; Butler, 1988 ; Fischer-Lichte, 2004, 2012; Sting, 2008, 2012). Proche du translanguaging (Williams, 1994; Garcia, 2009, 2012, 2014 ; Backledge \& Creese, 2010 ; Canagarajah, 2011 ; Lewis, Jones \& Baker, 2012), nous inscrivons l'acte de translangager (Aden, 2012 : 268) dans une pédagogie de la translangageance (Eschenauer, 2014, 2017), qui prend appui sur la plasticité neuronale et les dynamiques corporelles des langages pour développer la capacité à naviguer entre toutes les formes de langages et de langues. Nos recherches mettent en avant deux aspects fondamentaux des interactions plurilingues : la place des mécanismes d'empathie et le rôle de l'expérience esthétique (Dewey, 1934; Couchot, 2012; Schaeffer, 2015) qui permet “d'ancrer les savoirs abstraits dans une connaissance sensible et incorporée du monde. » (Aden, 2008, p.11). Mettant en lien les racines biologiques du langage (Maturana \& Varela, 1987) et les racines esthétiques des langages poétiques (Lecoq, 1997; Fischer-Lichte, 2004) nous avons ouvert la voie à une pédagogie enactive-performative des langues (Aden, 2017; Eschenauer, sous presse). Dans cet article, nous introduirons la notion de «translangager » dans le paradigme de l'enaction, puis nous présenterons l'approche performative qui nous permet de mettre en œuvre une pédagogie que nous nommons enactive-performative. Nous illustrerons ensuite notre modèle du translangager avec l'exemple d'une expérimentation dans une classe plurilingue et pluriculturelle. Nous montrerons comment les mécanismes d'empathie (kinesthésique, émotionnelle et cognitive) qui engagent toutes les formes de langage (corporel, esthétique, culturel, linguistique) tissées avec l'expérience esthétique, constituent l'essence même de la translangageance (Eschenauer, 2017). Nous verrons qu'une telle pédagogie entraîne une nouvelle configuration des relations humaines et du lien social.

\section{DU PLURILINGUISME, COMPÉTENCE COMMUNICATIVE, À LA TRANSLANGAGEANCE, INTERACTION ENCORPORÉE ${ }^{1}$}

\footnotetext{
${ }^{1}$ Le terme encorporé (Eschenauer, 2014a, 2017) traduit embodied. « La traduction usuelle « incarné » pour embodied a une connotation qui peut porter à confusion, et qui fait allusion à la chair plus qu'au corps dans son ensemble. Embodied comprend non seulement les parties corporelles externes visibles mais également tout le système nerveux, centre des émotions, qui est relié au cerveau et est à la base de la perception sensorimotrice. Incorporé, également employé pour traduire ce terme, est à mon sens plus réducteur qu'encorporé. «In- » signifie dans, à l'intérieur, alors que « en- » exprime un phénomène en mouvement intérieur/extérieur dans le corps (du dehors vers le dedans, à l'intérieur et de l'intérieur vers l'extérieur). J'entends par langage encorporé le fait que les langues sont une partie de notre matrice langagière. À ce titre, elles sont inscrites dans nos corps en mouvement et s'impriment/s'expriment au travers d'eux » (Eschenauer, 2017, p.11).
} 
L'éthique plurilingue et pluriculturelle introduite en 2001 dans le CECRL constitue une avancée majeure pour la didactique des langues. Cependant, plus de quinze ans après la publication de ce texte, force est de constater qu'en France, les expériences de terrain permettant aux langues de se rencontrer sont encore très rares. On en trouve certaines dans les dispositifs bilangues mais, dans la plupart des cas, ils ne mettent en relation que les langues vivantes étrangères ou dans des d'approches plurielles mais qui sont dissociées des programmes scolaires. Si notre approche présente des liens avec la didactique intégrée, il n'existe pas, à notre connaissance, de dispositif associant toutes les langues de l'école apprises par une cohorte d'élèves et impliquant dans le même temps les langues des familles de ces apprenants dans une pédagogie transdisciplinaire. C'est le défi que nous avons relevé et qui nous amène à passer de la juxtaposition des langues et de la linéarité des programmes à une approche complexe et dynamique. Nous nous appuyons sur le paradigme enactif, que Varela explicite ainsi: «nous proposons (...) le terme d'enaction dans le but de souligner la conviction croissante selon laquelle la cognition, loin d'être un monde prédonné, est l'avènement conjoint d'un monde et d'un esprit à partir de l'histoire des diverses actions qu'accomplit un être dans le monde » (Varela, Thompson \& Roch., version anglaise, 1993, p. 35). Ainsi le langage ne reflète pas un monde, mais il créé le monde que nous partageons dans l'action. L'ancrage de notre compréhension des langues et des langages dans leur définition varélienne (Aden, 2017) nous invite à revisiter les notions de «plurilinguisme» et de «communication» depuis des fondements phénoménologiques, neurologiques, socio-linguistiques. Tout au long de sa carrière scientifique, Varela a en effet mis en résonance la philosophie et les sciences exactes. Il a initié une «révolution » avec son programme neurophénoménologique $(1996,1999)$ en plaçant la subjectivité au coeur des sciences dures. Il l'exprime ainsi dans l'une de ses dernières interviews : «La méthode scientifique exige que la recherche soit rendue publique, ouverte à vérification. Or, l'expérience subjective est, par définition, privée et n'est donc accessible qu'à l'expérience personnelle. (...) Les scientifiques devraient créer une nouvelle science de la conscience où la subjectivité n'est ni réduite, ni occultée, mais centrale. » (Varela, 2002, p. 163). La subjectivité est la manifestation de l'expérience sensible, incorporée. Pour Varela, «l'esprit n'est pas autre chose que le corps en mouvement. » (Id., p. 174).

\section{Le plurilinguisme enacté : encorporé, relié et situé}

Dans le paradigme de l'enaction, le langage est à la fois le résultat et le moyen d'un «couplage structurel », qui rend possibles les « interactions continues d'un système structurellement plastique au sein d'un environnement»(Varela, 1989 : p.64). Pour Varela, l'être humain est constitué dans le langage et par le langage qui émerge dans les interactions. Plus généralement, apprendre c'est se relier 
dans l'action avec les autres, et en se reliant, faire émerger du sens. Le sens n'est pas extérieur à nous, il émerge de nos interactions constantes avec les autres et avec leur connaissance, leurs perceptions, leurs actions. Les actions-perceptions partagées modifient l'organisation cognitive des individus en présence. Comme nous le rappelle Hélène Trocmé-Fabre : communiquer vient du latin co-munio : «je construis avec ». «Il s'agit d'une réalisation sociétale par acte de langage, regard ou geste, dans le but de construire un espace-temps commun » (Trocmé-Fabre, 2013, p.116). Dans son assertion enactive, la communication émerge dans des réseaux complexes qui relient nos perceptions, nos actions, nos émotions, nos langues dans différents contextes - c'est à dire, la famille, l'école, le quartier, le pays, les modes virtuels, etc... Dans les écoles françaises, les langues des familles sont généralement exclues et l'on conseille parfois aux parents de parler un français qu'ils maîtrisent mal à leurs enfants pour qu'ils s'intègrent mieux, si bien que les élèves éprouvent de la gêne, voire de la honte, à évoquer leurs langues. Dans une approche enactive de l'enseignement, les dispositifs valorisent nécessairement les langues des familles en tant qu'elles sont ontologiques, donc indissociable de la manière d'apprendre des élèves.

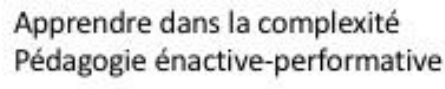

Déréalisation de l'autre:

Rupture des

mécanismes

d'empathie (Spitzer; Van Eersel)

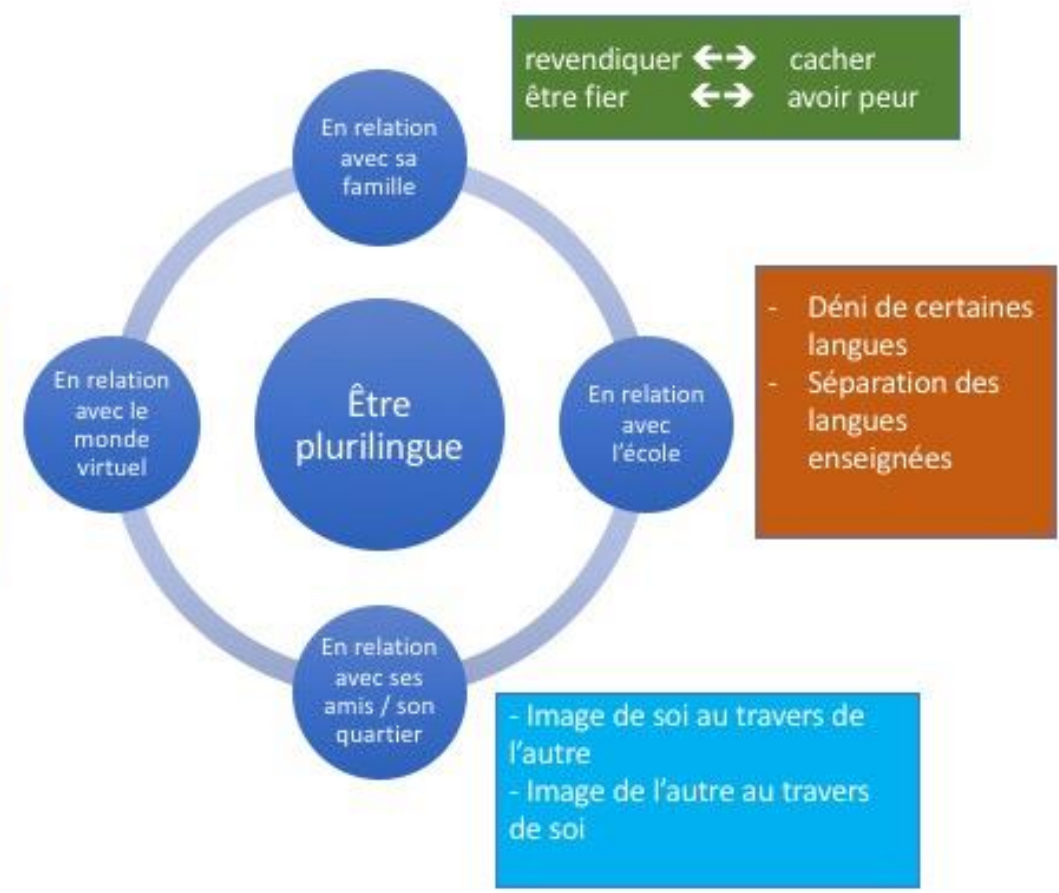

S'inspirant également de la notion de languaging proposée par Maturana et Varela (1994), Ofelia García s'intéresse plus particulièrement à « l'organisation autopoïétique du languaging dans les interactions sociales $^{2} \gg$ d'un point de vue sociopolitique (2014, p. 203). Bien que notre approche vise aussi une transformation des représentations sociales des langues, nous nous focalisons, pour notre part, sur les

\footnotetext{
2 "autopoietic organization of languaging across national, socio political, and social interactions"
} 
aspects cognitifs qui constituent un des « niveaux d'émergence spécifiques » (Varela, 2002, p. 175). En effet, Varela considérait que «l'une des grandes erreurs est de confondre les niveaux d'organisation. Ainsi l'autopoïèse décrit-elle le fonctionnement du niveau d'organisation cellulaire. Mais le système immunitaire, le cerveau, l'organisme humain ne sont pas autopoḯtiques. Ils possèdent d'autres caractéristiques d'organisation qui permettent leur émergence. » (Idem).

Nous nous focalisons donc sur l'organisation cognitive dans une conception holistique, incorporée et dynamique du translangager qui «dépend d'un monde inséparable de nos corps, de nos langages et de nos histoires culturelles - en d'autres termes, de notre corporéité »(Id., p. 210). Des recherches de plus en plus nombreuses dans le champ des neurosciences mettent en évidence la plasticité du cerveau capable de s'adapter à toutes les contraintes du corps dans son milieu. Cette plasticité neuronale concerne également le langage, comme le met en évidence la neurologue Ellen Bialystok dans ses études sur le bilinguisme. Ses résultats montrent que l'utilisation de plus d'une langue change la structure du cerveau mais pas forcément dans le sens attendu par les enseignants de langues. Par rapport aux monolingues, les compétences linguistiques des bilingues sont moins bonnes dans chacune de leurs langues $^{3}$, en revanche, ils montrent une plus grande neuroplasticité dans le contrôle exécutif quand il s'agit de faire des choix entre leurs deux langues et ils ont une meilleure capacité à inhiber les informations erronées. Les auteurs concluent leur étude par l'observation que le fait de porter son attention sur deux langues favorise une «meilleure performance cognitive » (Bialystok, Craik, and Luk, 2012, p. 241). Ces résultats rejoignent nos observations de terrain qui montrent que les dispositifs artistiques performatifs menés en plusieurs langues développent des attitudes d'empathie, des compétences cognitives psycho-sociales et une plus grande flexibilité mentale (Eschenauer, 2017) qui constituent le socle sur lequel se construisent les dimensions linguistiques de l'apprentissage (Aden \& Eschenauer, 2014). Bialystok \& ali. «proposent un modèle distribué dans lequel les deux langues sont utilisées en continu à des niveaux différents. Pour eux, les bilingues ne passent pas d'une langue à l'autre mais ils régulent l'activation conjointe des deux langues grâce aux fonctions exécutives ${ }^{4}$ qui influencent leurs choix linguistiques en fonction du contexte et des caractéristiques individuelles des sujets. » (in Aden \& Pavlovskaya, sous presse). Ainsi le passage d'une langue à une autre en lien avec l'apprentissage de nouvelles langues s'enracine dans l'expérience partagée corporellement, vécue dans des contextes spécifiques.

\section{Du translangager à la translangageance}

\footnotetext{
${ }^{3}$ moins de vocabulaire et un accès moindre aux éléments lexicaux.

${ }^{4}$ Processus cognitifs de base tels que l'attention, l'inhibition, la mémorisation ou la flexibilité.
} 
Il est nécessaire de préciser que « l'enaction n'est pas une théorie du langage, c'est un paradigme de la connaissance dans lequel le langage tient une place centrale car il constitue la sève qui nous relie aux autres, à la connaissance du monde et à la connaissance de soi. » (Aden, 2017, p. 9). Là où l'allemand et l'anglais n'utilisent qu'un seul mot, "Sprache/language”, la langue française fait une distinction importante entre les termes «langage » et «langue». La notion de langage chez Maturana et Varela est très large, elle décrit l'ensemble des capacités qui permettent d'agir ensemble. Le langage est incorporé car il résulte de comportements sensori-moteurs qui émergent dans le couplage social, au sein duquel il donne naissance à des sens partagés. Il englobe toutes les modalités de l'interaction (corporelle, émotionnelle, verbale et culturelle) et il ne se réduit donc pas à la verbalisation du sens (langues). Pour Varela et Maturana, la complexité et la diversité des languages (langues) constituent «l'épiphénomène d'un vaste système dynamique et complexe dont la fonction première n'est pas de transmettre de l'information, mais de comprendre le monde et d'agir ensemble dans le monde. » (1994, p. 241). « À l'intérieur du langage, les langues orales et écrites reflètent donc le niveau de précision et d'abstraction totalement unique dont notre espèce est capable » (Aden, 2017, p.8). Ainsi, nous considérons qu'apprendre de nouvelles langues ne consiste pas seulement à réorganiser des connaissances linguistiques de surface, mais que cela entraîne une reconfiguration dynamique de tout le système langagier dans l'action conjointe, et requiert une démarche holistique. Pour ce qui nous concerne, nous avons élargi et étayé la notion de translangager en y intégrant les répertoires kinesthésiques, sensoriels et émotionnels qui permettent aux élèves d'établir des liens entre tous les langages au-delà et en deçà du verbal (Aden, 2012). Nous intégrons par ailleurs l'ensemble des répertoires linguistiques des élèves pour éviter les ruptures identitaires, d'une part, et pour permettre aux élèves de trouver du sens dans l'apprentissage de nouvelles langues étrangères, d'autre part. Il ne s'agit pas d'ajouter des disciplines à des connaissances acquises, mais d'enrichir la biographie langagière (Krumm, 2003) des élèves.

Pour pouvoir aider les enfants à faire le travail de passage d'un monde à l'autre, les enseignants, comme les cliniciens, doivent modifier leurs positions intérieures et se sensibiliser à ce que vivent les enfants. Il ne s'agit pas en effet de mettre en place des modalités spécifiques d'apprentissage, ou d'introduire une quelconque discrimination, mais de servir de passeurs aux enfants, de leur donner envie d'atteindre une rive en s'appuyant sur celle qu'ils connaissent et qu'ils doivent apprendre à valoriser. (Moro, 2002, p. 63)

Alors que le verbe translangager exprime «l'acte dynamique de reliance à soi, aux autres et à l'environnement par lequel émergent en permanence des sens partagés entre les humains » (Aden, 2013, p. 115), la notion de translangageance permet de décrire le «processus d'émergence d'un langage commun qui fait sens, à travers toutes les formes de langages » (Eschenauer, 2017, p. 15) et elle inscrit également ce processus dans les durées biologiquement nécessaires à tout apprentissage des langues. «La notion de translangaeance comprend donc des compétences de bas niveau (translangageance hors contrôle) et de haut niveau (translangageance sous contrôle), qui s'opèrent dans 
un va-et-vient continu dès lors que les élèves peuvent allier un vécu expérientiel émotionnel et un vécu expérientiel réflexif, leur permettant d'accéder à un niveau de conscience de plus en plus fin de processus intra- et intersubjectifs » (Eschenauer 2017, p. 141-142). Ainsi, le «trans » du langager constitue-il l'interface entre intersubjectivité et subjectivité, il permet de construire une identité propre en lien avec des identités sociales multiples.

\section{LA PERFORMANCE TRANSLANGUES, UNE NOUVELLE PERSPECTIVE EN DIDACTIQUE DES LANGUES ?}

Il existe des propositions pédagogiques de plus en plus nombreuses s'appuyant sur le théâtre, le Drama ou d'autres pratiques artistiques. Manfred Schewe $(2011,2013)$ par exemple, défend une didactique performative des langue vivantes prenant en compte le champ de l'esthétique dans une approche basée sur le drama. Il propose de l'appliquer aux domaines scolaires des langues, de la littérature et de la culture. Dans le champ des sciences de l'éducation, Wolfgang Sting $(2012,2017)$ s'appuie également sur la notion de performativité qu'il explore dans une pédagogie transdisciplinaire. Au-delà du drama, il privilégie une approche phénoménologique et esthétique des apprentissages scolaires. Selon lui, la performance (Fischer-Lichte, 2012) permet de réduire le fossé entre l'inconnu et le familier au travers d'une approche expérientielle et esthétique - donc active, (inter)subjective, émotionnelle, sensorielle. Ces vécus performatifs de l'apprendre engagent aussi bien les corps en action que les échanges dialogiques.

Pour notre part, si nous nous inscrivons dans le courant performatif de Fischer-Lichte et de Wolfgang Sting adossés aux principes de la philosophie de l'enaction, nous y intégrons les dimensions translangues et transcultures (Aden \& Eschenauer, 2014) dans une "esthétique relationnelle" que (Bourriaud, 1998) définit ainsi :

\footnotetext{
« un 'matérialisme de la rencontre' (...) qui prend pour point de départ la contingence du monde, qui n'a ni origine, ni sens qui lui préexiste, ni Raison qui lui assignerait un but. Ainsi, l'essence de l'humanité est-elle purement transindividuelle, faite de liens qui unissent les individus entre eux dans des formes sociales qui sont toujours historiques. Il n'existe pas de 'fin de l'histoire' ni de 'fin de l'Art' possibles, puisque la partie se réengage en permanence, en fonction du contexte, c'est-à-dire en fonction des joueurs et du système qu'ils construisent ou critiquent. » (p. 16-17)
}

C'est pourquoi nous imaginons nos projets pédagogiques comme des "questionnements vivants" (living enquiries) (Irwin, LeBlanc, Ryu, Belliveau, 2017) menés en communautés de recherche et d'expériences dans toutes les langues des apprenants et des pédagogues : artistes, enseignants, chercheurs et médiateurs culturels qui élaborent ensemble des projets répondant aux besoins des élèves. Cela nous évite d'instrumentaliser l'Art et nous permet d'en préserver la nature fondamentalement émergente, contingente, esthétique et phénoménale. Les élèves font l'expérience du vécu d'acteur et de celui de spectateur, au travers de formes présentées en différentes langues. La partie dialogique qui 
accompagne nécessairement le vécu de spect-acteur (Boal, 2001) vise à déclencher une prise de conscience des dimensions esthétiques de la connaissance. Ainsi, nous intégrons les questionnements des élèves dans les objectifs de progressivité des programmes scolaires, tout en développant leur autonomie langagière au travers d'une expérience performative translangues.

\section{Liens entre performativité et translangageance}

Dans ce paragraphe, nous décrivons notre approche enactive-performative à partir des caractéristiques de la performativité telles que définies par Fischer-Lichte (2004, 2012). Autour du concept central d'émergence, nous retrouvons les fondements de l'enaction : la corporéité (embodied mind), le primat de l'action, l'expérience sensible (empathie), le couplage subjectivité/intersubjectivité. 


\begin{tabular}{|c|c|c|}
\hline & $\begin{array}{l}\text { Performance } \\
\text { Approche performative }\end{array}$ & $\begin{array}{c}\text { Translangageance } \\
\text { Approche enactive du translangager }\end{array}$ \\
\hline \multirow{2}{*}{ 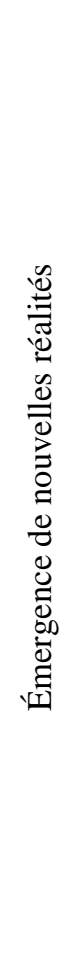 } & $\begin{array}{l}\text { La performance est émergente: } \\
\text { Comme toute relation humaine, elle se } \\
\text { déploie dans le moment de l'action } \\
\text { partagée entre les acteurs (au sens large : } \\
\text { ceux qui agissent) et les spectateurs (ceux } \\
\text { qui découvrent) }\end{array}$ & $\begin{array}{l}\text { La relation émerge dans l'acte de translangager à plusieurs } \\
\text { niveaux : } \\
\text { - émergence d'un sens partagé dans le moment de l'action } \\
\text { et dans un espace donné } \\
\text { - émergence de différents registres langagiers et du choix } \\
\text { des registres, toujours en fonction de la situation } \\
\text { - émergence de productions verbales et scéniques sans } \\
\text { préparation préalable }\end{array}$ \\
\hline & $\begin{array}{l}\text { Ce faisant, la performance constitue une } \\
\text { nouvelle réalité : dans la performance, } \\
\text { "l'acteur" n'est plus dans la seule } \\
\text { représentation d'un "comme-si" comme } \\
\text { dans l'interprétation d'un texte théâtral, } \\
\text { mais son jeu constitue en lui-même une } \\
\text { nouvelle réalité partagée avec le public. } \\
\text { Les frontières acteur-spectateur sont } \\
\text { dépassées par le lien qui se noue dans } \\
\text { l'émotionnalité et la sensorialité du vécu } \\
\text { partagé par leur "co-présence corporelle" } \\
\text { (leiblicheKo-Präsenz: Fischer-Lichte, } \\
\text { 2012, p. 54); }\end{array}$ & $\begin{array}{l}\text { Les interactions entre les langues et les cultures } \\
\text { créent des changements de perspectives et font naître de } \\
\text { nouvelles réalités : } \\
\text { - Les scénarios didactiques ne sont pas écrits en amont } \\
\text { par des pédagogues extérieurs à la classe. } \\
\text { - Les élèves ne reproduisent pas "à la manière de" } \\
\text { locuteurs natifs, mais ils "sont" des êtres qui vivent } \\
\text { plusieurs langues, dont la ou les langues des familles et } \\
\text { celles de l'école. } \\
\text { - Les textes sont les leurs, nourris parfois par des apports } \\
\text { littéraires ou d'autres genres artistiques. }\end{array}$ \\
\hline 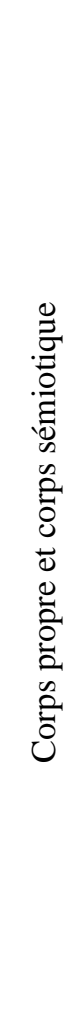 & $\begin{array}{l}\text { L'émergence du sens se réalise au travers } \\
\text { de la corporéité, dans la tension qui } \\
\text { réside entre le corps phénoménal (der } \\
\text { phänomenale Leib) - percevant, agissant } \\
\text { dans l'intime, porteur d'émotionalité - et } \\
\text { le corps sémiotique (der semiotische } \\
\text { Körper) - le corps visible par autrui. } \\
\text { Prenant l'exemple de la conception du jeu } \\
\text { d'acteur de Grotowski, Fischer-Lichte } \\
\text { précise que le corps Leib agit en tant } \\
\text { qu'esprit incarné "verkörperter Geist" } \\
\text { (embodied mind). (2004, p. 139) } \\
\text { En effet, ce que nous percevons, pensons, } \\
\text { ressentons etc., ne peut pas se dissocier de } \\
\text { nos gestes, de nos mouvements, de la } \\
\text { manière dont nous portons notre regard } \\
\text { sur quelque chose, de notre } \\
\text { positionnement dans l'espace. } \\
\text { Inversement, notre façon de nous } \\
\text { mouvoir, de nous positionner, de déplacer } \\
\text { notre regard est guidée par ce que nous } \\
\text { percevons au plus intime de nous-mêmes. } \\
\text { Le corps-Leibkörper est donc un } \\
\text { médiateur entre nous et le monde. }\end{array}$ & $\begin{array}{l}\text { - En translangageant, un équilibre s'opère entre le corps } \\
\text { et l'esprit biologiquement indissociables mais souvent } \\
\text { sollicités de manière disjointe à l'école. } \\
\text { - L'élève s'appuie à la fois sur son corps-Leib (celui qu'il } \\
\text { est) et sur son corps-Körper (celui qu'il a). Il/ Elle } \\
\text { perçoit de façon préconsciente son corps-Leib vivant, } \\
\text { ontologique (sensorialité, émotions, affect etc. : en } \\
\text { moyen haut allemand, "Lip" signifie de la même } \\
\text { manière la vie, 'Leben', et le corps, 'Leib'), et agit de } \\
\text { façon consciente avec son corps-Körper (le corps } \\
\text { anatomique et physiologique, celui que l'on voit de } \\
\text { l'extérieur, que l'on manipule). } \\
\text { - Cet équilibre Leibkörper émerge en translangeant avec } \\
\text { les autres élèves, les artistes, les enseignants. } \\
\text { - Les apprentissages cognitifs, intellectuels, réflexifs sont } \\
\text { reliés aux langues "étrangères" vécues dans la } \\
\text { performance (espritcorps). } \\
\text { - Les techniques du jeu d'acteur facilitent les } \\
\text { changements de perspective nécessaires au passage } \\
\text { d'une langue et d'une culture à l'autre car elles utilisent } \\
\text { les mécanismes d'empathie kinesthésique, émotionnelle } \\
\text { et cognitive. }\end{array}$ \\
\hline
\end{tabular}




\begin{tabular}{|c|c|c|}
\hline 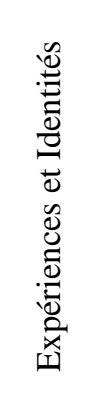 & $\begin{array}{l}\text { Par la performance, il ne s'agit pas } \\
\text { d'exprimer, de représenter) une identité (en } \\
\text { tant que réalité corporelle et sociale), mais } \\
\text { l'identité se constitue au travers du } \\
\text { leibköper co-agissant. En cela, la } \\
\text { performance est "auto-référentielle" et } \\
\text { génère une expérience esthétique chez les } \\
\text { participants. (Fischer-Lichte, 2004, p. 245) }\end{array}$ & $\begin{array}{l}\text { - La diversité des identités et des expériences des élèves } \\
\text { constitue le matériau vivant à partir duquel les artistes, les } \\
\text { enseignants, les élèves co-construisent les scénarios } \\
\text { pédagogiques (en classe ou en ateliers artistiques). } \\
\text { - Ces identités se transforment et s'enrichissent dans le } \\
\text { temps dans l'expérience partagée. } \\
\text { - Le regard esthétique que les artistes posent sur le monde } \\
\text { et sur les élèves et leurs langues a le pouvoir de les émouvoir } \\
\text { et de les transformer : leurs repères changent et ils se } \\
\text { perçoivent sous un jour inédit et bienveillant. }\end{array}$ \\
\hline 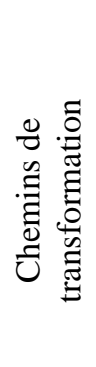 & $\begin{array}{l}\text { ainsi, la performance représente “une } \\
\text { force transformatoire" (2012, p.113), qui } \\
\text { change aussi bien les personnes que } \\
\text { l'espace et les éléments du contexte. Celle- } \\
\text { ci est appuyée par des éléments scéniques, } \\
\text { en particulier l'espace et son utilisation, la } \\
\text { corporéité, l'intensivité vocale et le timbre } \\
\text { de la voix, le rythme etc. (Lange in Koch } \\
\text { et al., 2003, p. } 220)\end{array}$ & $\begin{array}{l}\text { Par la performance, l'apprentissage des langues est perçu } \\
\text { non pas comme "l'acquisition de savoirs" ou de } \\
\text { "compétences en langues", mais comme des "chemins de } \\
\text { transformation" (Varela, in Trocmé-Fabre, 1995). La prise } \\
\text { de conscience du souffle, du rythme, les variations de la } \\
\text { pratique langagière dans le mouvement et dans l'espace, } \\
\text { les jeux corporels participent de cet apprentissage } \\
\text { dynamique et encorporé. }\end{array}$ \\
\hline
\end{tabular}

Cette mise en regard de la performativité et de la translangageance révèle l'hyper-complexité du langage humain qui émerge dans un équilibre subtil entre le corps, l'esprit et la conscience réflexive comme Sandrine Eschenauer l'a formalisé dans sa recherche doctorale (2017). En effet, "les mécanismes d'empathie favorisent le passage d'une langue et d'une culture à l'autre, et réciproquement la capacité à translangager aide au développement d'attitudes empathiques". Par ailleurs, "l'expérience esthétique vécue au travers d'une approche performative (théâtre) dans toutes les langues des élèves semble directement corrélée au développement des capacités empathiques altruistes.”

Les dimensions esthétique, préverbale, préconsciente du translangager constituent le socle de compétences psychosociales telles que l'acceptation de l'autre et de soi-même ou la capacité à l'écoute empathique. Ces compétences sont basées sur des fonctions de contrôle indispensables à tout apprentissage : l'attention, l'inhibition, la mémorisation ou la flexibilité mentale.

\section{UN EXEMPLE DE PROJET ENACTIF-PERFORMATIF DANS UN ENVIRONNEMENT PLURILINGUE}

L'étude longitudinale intitulée AiLES (Arts in Language Education for an Empathic Society) s'est déroulée dans un collège pluriculturel d'un quartier défavorisé de la région parisienne. Notre problématique initiale de recherche était la suivante : les pratiques artistiques translangues, notamment le théâtre, qui s'appuie sur les émotions et les mécanismes d'empathie tout en les développant, peuventelles favoriser le développement de stratégies de médiations langagières chez les élèves et faciliter les apprentissages conjoints d'allemand et d'anglais ? Dans le même temps, la capacité croissante des 
élèves à se mettre en empathie avec autrui pourrait-elle les aider dans leur jeu d'acteur plurilingue, et dans leur capacité à passer d'un langage à l'autre?

Afin de la vérifier, nous avons suivi durant quatre ans (2011-2015) une même classe dite bilangue : les élèves y apprenaient deux langues étrangères dès l'entrée en $6 \mathrm{e}^{5}$ (l'allemand et l'anglais). Dans cette classe de 20 élèves, plus de 16 langues étaient parlées et/ou comprises.

\section{Le dispositif : des ateliers artistiques translangues inclus dans les enseignements de LVE}

Au-delà des objectifs linguistiques et culturels propres à la didactique des LVE, l'ensemble de l'équipe pédagogique et artistique a fixé des objectifs émotionnels, esthétiques, métacognitifs etc., nécessaires pour mettre la relation au cœur du dispositif holistique. Il s'agissait de créer du lien entre les disciplines scolaires et les pratiques performatives ; mais aussi entre l'école, les familles, les enseignants, les artistes et les élèves etc.

Des comédiennes sont intervenues à hauteur d'une vingtaine d'heures d'ateliers par an, intégrés aux cours de langues. Elles ont parfois été accompagnées d'un photographe, d'un cadreur, ou d'un danseur. Chacun s'exprimait dans sa langue (allemand, anglais, hébreux, français). Ils n'avaient jamais d'objectifs académiques, n'adaptaient pas leur discours, ni même leur débit pour les élèves. Ils menaient l'atelier dans l'action, en montrant, en créant avec les élèves, en s'appuyant sur les mécanismes d'empathie kinesthésique et émotionnelle : gestes, mimiques, variations vocales, etc. Dans certaines situations, ces médiations corporelles étaient complétées de médiations linguistiques, afin d'éviter les ruptures dans le travail théâtral. D'abord portées par les adultes, ces compétences ont été progressivement prises en main par les élèves qui ont déployé des stratégies de collaboration et d'entraide.

\section{Mise en auvre pédagogique du translangager}

Les enseignantes intégraient les séances de théâtre à leurs objectifs pédagogiques et, à l'inverse, elles faisaient part de leurs objectifs aux comédiennes qui pouvaient s'en inspirer. Quand des opportunités émergeaient, les enseignantes de langues construisaient des cours ensemble ce qui leur permettait de mettre en évidence les ressemblances et différences entre les langues, notamment par des activités d'observation réfléchie des langues (Kervran, 2006). Elles étudiaient les mêmes supports dans des versions bilingues ou traitaient un sujet à partir de documents complémentaires dans des langues différentes. Progressivement, les élèves ne se concentraient plus sur les langues en tant qu'objet

\footnotetext{
${ }^{5}$ 6. Klasse en Allemagne. En France, l'école élémentaire dure 5 ans, et le collège débute avec la 6è.
} 
d'apprentissage, mais les langues devenaient pour eux le moyen d'entrer dans la relation pour partager des idées, des actions. En témoignent ces propos :

«Parfois ils utilisent une langue et ils ne s'en rendent même pas compte (enseignante d'anglais)", ou s'ils ne trouvent pas le mot, "je vois qu'ils cherchent, ils cherchent et (...) ils le disent en allemand ; ils sont très conscients du fait qu'ils le disent en allemand, mais ça pose pas de problème. Ils continuent la phrase quand même, ils répondent en anglais (comédienne anglaise) » (Extrait de thèse S. Eschenauer, 2017, p. 427)

Ce désir d'apprendre et de partager les langues, le degré d'investissement, de solidarité, et de créativité des élèves, se sont accrus en fin de troisième année d'expérimentation, lorsque les élèves ont été touchés par les réactions émotionnelles du public durant leur performance finale. Ils ont alors souhaité travailler la dernière année sur une pièce de théâtre écrite plutôt que sur leurs improvisations. Lutz Hübner, dramaturge et metteur en scène contemporain allemand connu pour son engagement auprès des jeunes, a accepté de s'engager avec la communauté. Suite à une rencontre-discussion avec la classe, il a co-écrit une pièce germano-anglaise avec eux à partir de questionnaires sur les personnages que les élèves aimeraient incarner, et d'ateliers d'improvisations autour de ces sujets. La pièce qu'ils ont choisi d'intituler "Simply the Best" leur donnait la parole sur leur vie dans leur quartier, sur leurs désirs, leurs espoirs, leurs passions, leurs révoltes. Avec l'aide de leurs parents, les élèves y ont introduit des dialogues dans leurs langues familiales, à des moments-clé de la pièce fortement empreints d'émotions. Le thème « être libre, s'adapter, se rebeller » était également celui de la rencontre européenne annuelle du théâtre scolaire en langues vivantes de Turin, Lingue in scena, à laquelle ils ont eu la chance de pouvoir participer.

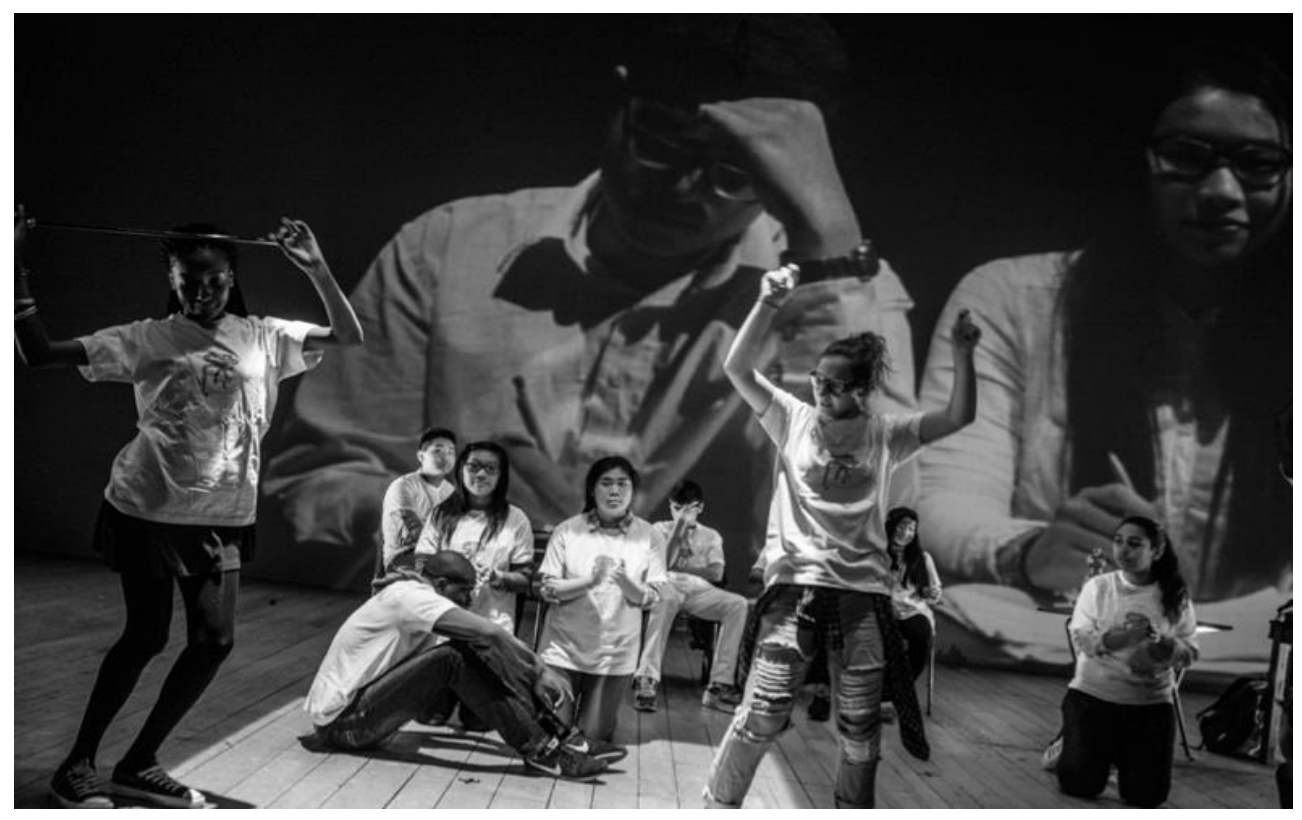

Figure 2 - Création commune, vers l'esthétique des élèves. Représentation de la pièce "Simply The Best" Lors du festival européen Lingue in Scena, Turin, Mai 2015 @photo Fred Furgol 


\section{Résultats}

Nous relevons ici quelques-uns des résultats parmi les plus significatifs obtenus à partir du croisement de données qualitative et quantitatives (Observations, entretiens d'explicitation, tests psychométriques) traitées avec le logiciel Nvivo.

a) Les élèves ont progressivement pris conscience de leur capacité à translangager, ils ont développé une plus grande confiance en eux, sont devenus plus créatifs dans leur expression et ils ont affiné leur maîtrise dans chaque langue. Nous notons que certains élèves ont exprimé le désir d'apprendre leurs langues familiales ou de nouvelles langues.

b) L'utilisation simultanée de plusieurs langues dans les ateliers artistiques, loin de rendre plus difficiles les apprentissages, relie les élèves à un vécu expérientiel, émotionnel et prévient des inhibitions d'ordre linguistique "qui ne sont jamais évoquées comme un obstacle" (Eschenauer, 2017, p. 377).

EnsAll. : Et ils sont très à l'aise entre eux en tous cas pour passer d'une langue l'autre. Ils comprennent vite la langue, l'allemand, l'anglais (...) ils arrivent, ils ont cette faculté de pouvoir restituer avec exactitude ce qui a été dit dans l'autre langue. Mais sans trop réfléchir (...) C'est une technique qu'ils ont acquise. (Ibid.)

Parfois, ces passages d'un registre langagier (y compris d'une langue à l'autre) sont automatisés, donc, de nouveau, inconscients. L'évolution de Chelsea montre bien les processus transformatoires à l'œuvre dans une telle approche. Cette élève est passé d'un rejet de l'Allemand en 5e à une grande facilité à passer de l'anglais à l'allemand sans même s'en rendre compte. Dans l'extrait ci-dessous, on lui demande en quelle langue elle dit ses répliques dans la pièce

Chelsea : Moi je dis, heu : ich bin Chelsea et je dis, heu, que j'adore, heu, le RnB et la danse Soul. Chercheure : Et ça tu le dis dans quelle langue?

Chelsea : Je le dis en anglais. Oui.

(Entretien d'explicitation, élève $<$ Chelsea $>$, 26/01/2015, lignes 61-64)

Elle évoque une phrase en allemand, pensant parler anglais. Son rôle, en réalité, est bien en allemand. Elle se remémore des souvenirs chargés d'émotions positives dans la peau de son personnage qu'elle a créé, dont elle est fière. Elle associe alors les paroles de son personnage à la langue anglaise qu'elle privilégie et qui la fait rêver. "À la manière des enfants bilingues, elle change de registre sans prendre conscience qu'elle le fait, car c'est le sens de ce qui est en train de se produire qui lui importe avant tout. Ses productions orales et écrites en allemand s'enrichissent et se complexifient dès lors qu'elle s'implique dans ses rôles". (Eschenauer, 2017, p. 452). 
c) L'attitude respectueuse, curieuse et bienveillante des adultes qui vivent le plurilinguisme comme une norme a eu un impact décisif sur la construction identitaire des élèves et leur sentiment d'efficacité personnelle : reconnus dans leurs singularités, ils vivent plus sereinement la diversité linguistique et culturelle de leur classe, comme l'atteste l'enseignante d'anglais.

EnsAng. : Et ehm mais ça ne lui posait pas de problèmes d'entendre (la comédienne allemande) me parler en allemand, moi qui lui ai répondu en anglais et qui en même temps s'adresse à $<$ Chelsea $>$ pour qu'elle comprenne (-) tu vois ? Je je crois qu'il y a aussi ce contexte particulier où il y a la coexistence des deux langues simultanées et euh (-) on se parle les deux langues sans aucun problème et les gamins sentent qu'on comprend aussi les deux langues. Et donc eux-même pff parlent les deux langues (-) et commencent à utiliser les deux langues euh (-) tu vois en avertissant même si je leur euh (-) dans le contexte de euh du (--) du projet si je leur parle en anglais et qu'ils me répondent en allemand, c'est normal. (Entretien, $13: 06-14: 09$, Extrait thèse Eschenauer, 2017)

d) Le principal résultat de l'étude est la corrélation significative et équilibrée entre le développement des attitudes d'empathie (émotionnelle, kinesthésique et cognitive), l'intensification de l'expérience esthétique et l'approfondissement de la capacité à translangager. La flexibilité mentale semble avoir la fonction de contrôle la plus entraînée dans ce dispositif.

\begin{abstract}
La flexibilité mentale est travaillée à la fois dans les processus performatifs/ créatifs, mais également dans les passage d'une langue à l'autre et dans les processus ego-/hétérocentrés propres à l'empathie. La langue est un moyen de la relation et non plus l'objet en soi de l'apprentissage. (...) Pour les élèves, cette flexibilité sensible et posturale est un levier de la flexibilité linguistique (capacité à passer d'une langue à l'autre). (Ils) « s'adaptent » plus facilement à de grandes exigences (Eschenauer, 2017, p. 447).
\end{abstract}

\title{
Conclusion
}

Le projet AiLES s'inscrit dans les travaux de notre équipe de recherche et plusieurs études en cours viennent prolonger notre chemin de réflexion et d'exploration de cette approche pédagogique qui implique un changement de perspective dans la formation des enseignants ; il ne s'agit pas de les “former" à l'utilisation de nouveaux outils mais de les aider à déployer leur créativité individuelle, à développer une attitude empathique et la capacité à se questionner et de coopérer en fonction des objectifs didactiques qu'ils se sont fixés.

Nos recherches mettent au jour les leviers constitués par la prise en compte et la valorisation de toutes les langues des élèves pour accueillir de nouvelles langues et le rôle de l'expérience esthétique dans le développement des fonctions cognitives et des attitudes propices au désir d'apprendre. Il s'agit d'une approche compatible avec les cadres institutionnels puisque l'enaction est un paradigme qui part de l'expérience vécue, indissociable de l'histoire personnelle et professionnelle des enseignants partagée avec leurs élèves.

C‘est bien l'épistémologie relationnelle de l'enaction que nous mettons au cœur de la translangeance. Nous ne prônons pas seulement une didactique intégrée de toutes les langues mais une transformation 
sociale des mentalités afin que la diversité, qui est une loi du Vivant (Jacob, 1970), soit reconnue et valorisée comme une norme à l'école. Le travail conjoint des sociolinguistes, des didacticiens de toutes les disciplines, des pédagogues et des artistes contribue à ce projet humaniste qui vise un équilibre social inédit qui met en avant la collaboration et l'empathie altruiste.

\section{Bibliographie}

ADEN, Joëlle (2017) : «Developing empathy through theatre : a transcultural perspective in Second Language Education ». In : Schewe, M \& Crutchfield, J (Eds.), Going Performative in Intercultural Education. International contexts - theoretical perspectives - models of practice. Bristol : Multilingual Matters.

ADEN, Joëlle (2017) : «Langues et langage dans un paradigme enactif », Recherches en didactique des langues et des cultures [En ligne], 14-1 |2017, mis en ligne le 19 décembre 2016, consulté le 5 mai 2017. URL : http://rdlc.revues.org/1085

ADEN, Joëlle (2016) : "Créer, innover par le théâtre : pour une pédagogie enactive des langues." Chapitre 6. In I. Puozzo (dir.). La créativité en éducation et en formation Perspectives théoriques et pratiques. Bruxelles : De Boeck. (pp. 105-117).

ADEN, Joëlle (2012) : «La médiation linguistique au fondement du sens partagé : vers un paradigme de l'enaction en didactique des langues ». In Aden, J. et Weissmann, D. (dir.): La médiation linguistique : entre traduction et enseignement des langues vivantes. Paris : ELA n ${ }^{\circ} 167$, pp. 267-284.

ADEN, Joëlle (2010) : L'empathie, socle de la reliance en didactique des langues-cultures. In J. Aden, T. Grimshaw, H. Penz, (Eds..). Teaching Language and Culture in an Era of Complexity : Interdisciplinary Approaches for an Interrelated World. Bruxelles : Peter Lang, Coll.Gram-R. (pp. 2344).

ADEN, Joëlle \& PAVLOSKAYA, Maria (in press) : Translanguaging and language creativity in Drama Staging". In : G. Mazzaferro (Ed.), Translanguaging as Everyday Practice, Multilingual Matters.

ADEN, Joëlle \& ESCHENAUER, Sandrine (2014) : « Théâtre et empathie en classe bilangue : didactiser l'émergence ». In : Spanghero-Gaillard, N. Et Garnier, E. (dir.) : Pratiques théâtrales en classes de Langues. Les Langues Modernes, $\mathrm{n}^{\circ}$ 4/2014, 69-77.

AUSTIN, John L. (1962/1970) : Quand dire, c'est faire, Paris, Folio Essais.

BAKER, Colin, JONES, Bryn \& LEWIS, Gwyn (2012) : « Translanguaging: origins and development from school to street and beyond ». In : Educational Research and Evaluation, 18-7, pp. 655-670.

BIALYSTOK, Ellen, CRAIK, Fergus \& LUK, Gigi (2012) : «Bilingualism: consequences for mind and brain ». In : Trends in Cognitive Sciences April 2012, Vol. 16, No. 4, pp. 240-250.

BUTLER, Judith (1988): "Performative Acts and Gender Constitution: an Essay in Phenomenology and Feminist Theory" in Theatre Journal, Vol. 40, No. 4 (Dec., 1988), The Johns Hopkins University Press, pp. 519-531

BROOK, Peter (1977) : L’Espace vide, Paris, Éditions du Seuil. 
CANAGARAJAH, Suresh (2011) : "Codemeshing in Academic Writing: Identifying Teachable Strategies of Translanguaging ». In : The Modern Language Jounal, 95, pp. 401-417.

COUCHOT, Edmond (2012) : La nature de l'art, Paris, Hermann.

CREESE, Angela \& BLACKLEDGE, Adrian (2010): « Translanguaging in the Bilingual Classroom: A Pedagogy for Learning and Teaching? » The Modern Language Journal, 94, pp.103-115.

DEWEY, John (1934/2005) : L'art comme expérience, Paris, Folio Essais

ESCHENAUER, Sandrine (in press) : «Translanguaging and Empathy: effects of performative approach to language learning ", in Fleiner, M. \& Mentz, O.: The Arts in Language Teaching. International Perspectives : Performative - Aesthetic - Transversal - Europa lernen. Perspektiven für eine Didaktik europäischer Kulturstudien, LIT Verlag (Berlin, Vienna, Zurich, London) - Expected publication date : 2018.

ESCHENAUER, Sandrine (2017) : Médiations langagières dans une pédagogie enactive au collège. Étude longitudinale des liens entre les phénomènes de translangageance, d'empathie et d'expérience esthétique et leur impact cognitif dans un enseignement performatif des langues vivantes, thèse de doctorat en Sciences du langage, Université Paris-Est (UPE), Créteil, France

ESCHENAUER, Sandrine (2014) : «Faire corps avec ses langues. Théâtre et didactique : vers une définition de la translangageance ». In Actes du colloque Langues en mouvement, Languages in Motion, Nantes : E-Crini, $\mathrm{N}^{\circ} 6$, 2014, ISSN 1760-4753, p.1-24 [En ligne : http://www.crini.univnantes.fr/actes-de-colloque-langues-en-mouvement-didactique-des-langues-et-pratiques-artistiques-1145716.kjsp]

FISCHER-LICHTE, Erika (2004/2014) : Ästhetik des Performativen. Frankfurt-am-Main, FischerVerlag

FISCHER-LICHTE, Erika (2012) : Performativität. Eine Einführung, Bielefeld, Transkript.

GARCÍA, Ofelia \& WEI, Li (2014) : Translanguaging. Language, Bilingualism and Education, London et al., Palgrave Macmillan.

GARCÍA, Ofelia (2012) : « Theorizing translanguaging for educators ». In : C. Celic \& K. Seltzer (Eds), Translanguaging : A CUNY-NYSIEB guide for educators (pp.1-6). New York : Cuny-Nysieb.

GARCÍA, Ofelia (2009) : «Education, multilingualism and translanguaging in the 21st century ». In : Multilingual Education for Social Justice : Globalising the local, ed. by Ajit Mohanty, Minati Panda, Robert Phillipson, Tove Skutnabb-Kangas. New Delhi : Orient Blackswan (former Orient Longman), pp. $128-145$

GOFFMAN, Erving (1974) : Les rites d'interaction, Lonrai, Éditions de Minuit, Collection Le Sens Commun.

HUSSERL, Edmund (1921-1928/1973): Texte aus dem Nachlass. Zweiter Teil: 1921-1928: Zur Phänomenologie der Intersubjektivität. Texte aus dem Nachlaß, 2. Teil : 1921-1928. Herausgegeben von Iso Kern (@1973), The Hague, Netherlands

IMMORDINO-YANG, Mary Helen (2015) : Emotions, Learning, and the Brain. Exploring the Educational Implications of Affective Neuroscience, New York, London, W.W. Norton \& Company 
KRUMM, Hans Jürgen (2013) : «Multilingualism and identity, What linguistic biographies of migrants can tell us ». In : Siemund, Gogolin \& al. : Multilingualism and Language Diversity in Urban Areas: Acquisition, identities, space, education, Hamburg, John Benjamins Publishing Company, pp. 165-176.

LACHAUX, Jean-Philippe (2011) : Le cerveau attentif. Contrôle, maîtrise et Lâcher-Prise. Paris, Odile Jacob.

LECOQ, Jacques (1997) : Le corps poétique : un enseignement de la création théâtrale, Paris, Actes sud-Papiers.

MERLEAU-PONTY, Maurice (1945) : Phénoménologie de la perception, Paris, Gallimard.

SCHAEFFER, Jean-Marie (2015) : L'expérience esthétique, Paris, Gallimard Essais.

SCHECHNER, Richard (2002) : Performance Studies, London, New-York, Routledge.

SCHEWE, Manfred (2011) : «Die Welt auch im fremdsprachlichen Unterricht immer wieder neu verzaubern ». In : Küppers, Almut; Schmidt, Torben \& Walter, Maik (Hrsg.), Inszenierungen im Fremdsprachenunterricht. Kempten : Diesterweg, pp. 20-31.

SEARLE, John (1969/1972) : Les actes de langage, Paris : Hermann.

STING, Wolfgang et al. (2017): “Die Fremdheit der Schweine. Eine Fallstudie zum Wechselspiel von ästhetischer und diskursiver Praxis im Kontext kultureller Bildung"; in Konietzko, Kuschel \& Reinwand-Weiss (Hrsg.) Von Mythen zu Erkenntnissen? Empirische Forschung in der Kulturellen Bildung. Schriftenreihe Kulturelle Bildung, vol. 56, München 2017, pp. 95-111

STING, Wolfgang (2012) : «Performative Praxen und Theaterpädagogik». In : STING et al. : TUSCH : Poetiken des Theatermachens, Werkbuch für Theater und Schule, Bobingen, Kessler Druck und Medien/ Kopaed, pp. 119 - 126.

THIRIOUX, Bérangère, MERCIER, M. R., BLANKE, Olaf \& BERTHOZ, Alain (2014b): « The cognitive and neural Time Course of Empathy and Sympathy: an electrical neuroimaging study of selfother interaction ». In : Neuroscience 267 (2014), pp. 286-306.

VARELA, Francisco (2017) : Le cercle créateur. Écrits (1976-2001). Sous la responsabilité de Michel Bitbol. Avec le concours d'Amy Cohen-Varela, Jean-Pierre Dupuy et Jean Petitot. Paris, Éditions du Seuil.

VARELA, F. (1999) Quatre phares pour l'avenir des sciences cognitives, Revue Théorie Littérature Enseignement, $\mathrm{n}^{\circ} 17, \mathrm{pp} .7-21$.

VARELA, Francisco (1996) : «Neurophenomenology. A Methodological Remedy for the Hard Problem ». In : Journal of Consciousness Studies, 3, No. 4, 1996, pp. 330-349.

VARELA, Francisco (1989) : Autonomie et connaissance. Essai sur le Vivant, Paris, Edition Seuil.

VARELA, Francisco, THOMPSON, Evan \& ROSCH, Eleanor (1993) : L'inscription corporelle de l'esprit : sciences cognitives et expérience humaine, Paris, Seuil. 